This item was submitted to Loughborough's Research Repository by the author.

Items in Figshare are protected by copyright, with all rights reserved, unless otherwise indicated.

\title{
Acoustic black holes: recent developments in the theory and applications
}

PLEASE CITE THE PUBLISHED VERSION

http://dx.doi.org/10.1109/TUFFC.2014.3036

PUBLISHER

(c) IEEE

VERSION

AM (Accepted Manuscript)

LICENCE

CC BY-NC-ND 4.0

REPOSITORY RECORD

Krylov, Victor V.. 2014. "Acoustic Black Holes: Recent Developments in the Theory and Applications". figshare. https://hdl.handle.net/2134/15536. 
This item was submitted to Loughborough's Institutional Repository (https://dspace.lboro.ac.uk/) by the author and is made available under the following Creative Commons Licence conditions.

\section{creative
commons}

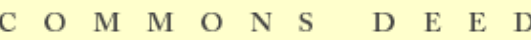

Attribution-NonCommercial-NoDerivs 2.5

You are free:

- to copy, distribute, display, and perform the work

Under the following conditions:

Attribution. You must attribute the work in the manner specified b the author or licensor.

Noncommercial. You may not use this work for commercial purposes.

No Derivative Works. You may not alter, transform, or build upon this work.

- For any reuse or distribution, you must make clear to others the license terms of this work.

- Any of these conditions can be waived if you get permission from the copyright holder.

Your fair use and other rights are in no way affected by the above.

This is a human-readable summary of the Leqal Code (the full license).

\section{Disclaimer 만}

For the full text of this licence, please go to: http://creativecommons.org/licenses/by-nc-nd/2.5/ 


\title{
Acoustic Black Holes: Recent Developments
}

\section{in the Theory and Applications}

\author{
Victor V. Krylov \\ Department of Aeronautical and Automotive Engineering, \\ Loughborough University, \\ Loughborough, Leicestershire LE11 3TU, UK \\ e-mail:V.V.Krylov@lboro.ac.uk
}

\begin{abstract}
Acoustic black holes' are relatively new physical objects that have been introduced and investigated mainly during the last decade. They can absorb almost $100 \%$ of the incident wave energy. And this makes them very attractive for such traditional engineering applications as vibration damping in different engineering structures and sound absorption in gases and liquids. They could be useful also for some ultrasonic devices using Lamb wave propagation to provide anechoic termination for such waves. So far, acoustic black holes have been investigated mainly for flexural waves in thin plates for which the required gradual changes in local wave velocity with distance can be easily achieved by changing the plate local thickness. The present paper provides a brief review of the theory of acoustic black holes, including their comparison with 'optic black holes' introduced about three years ago. Review is also given of the recent experimental work carried out at
\end{abstract}


Loughborough University on damping structural vibrations using the acoustic black hole effect. This is followed by the discussion on potential applications of the acoustic black hole effect for sound absorption in air.

Keyword-acoustic black holes; flexural waves; Lamb waves; vibration damping; sound absorption

\section{Introduction}

During the last decade, new physical objects have been proposed and investigated 'acoustic black holes' that can absorb almost $100 \%$ of the incident wave energy (see e.g. [15]). The main principle of operation of the acoustic black holes is based on a linear or higher order power-law-type decrease in velocity of the incident wave with propagation distance to almost zero accompanied by efficient energy absorption in the area of low velocity via small pieces of inserted absorbing materials. So far, this effect has been investigated mainly for flexural waves in thin plates (anti-symmetric Lamb modes of the lowest order) for which the required gradual reduction in wave velocity with distance can be easily achieved by changing the plate's local thickness according to a power law, with the power-law exponent being equal or larger than two. This principle has been applied to achieve efficient damping of flexural waves in plate-like structures using both one-dimensional 'acoustic black holes' (power-law wedges with their sharp edges covered by narrow strips of absorbing materials) and twodimensional 'acoustic black holes' (power-law-profiled indentations (pits) with small pieces of absorbing materials attached in the middle).

Recently, the above-mentioned basic principle of power-law reduction in velocity accompanied by efficient energy absorption in the area of low wave velocity has been reinvented in optics to implement the so-called 'optic black holes' [6, 7]. 
In the present paper, the theory of acoustic black holes is briefly discussed, and a comparative analysis of acoustic and optic black holes is given. The review is also given of some recent mainly experimental investigations carried out at Loughborough University.

There are still very few investigations of acoustic black holes for absorption of sound in gases and liquids. The possibilities of development of such acoustic black holes that could be used for sound absorption and traditional noise control are discussed as well.

\section{Theoretical background}

\section{A. Effect of zero reflection for waves in inhomogeneous media}

Let us first consider the 'effect of zero reflection' that can take place for waves of arbitrary physical nature propagating in inhomogeneous media with some specific types of variation of wave velocity with propagation distance. Considering for simplicity a one-dimensional wave propagation characterised by the distance $x$ in an ideal medium with power-law dependence of wave velocity $c$ on $x$ as $c=a x^{n}$, where $n$ is a positive rational number and $a$ is a constant, one can express the geometrical acoustics solution for the complex amplitude $U(x)$ of a wave propagating from any arbitrary point $x$ towards zero point (where $c=0$ ) as

$$
U(x)=A(x) e^{i \Phi(x)},
$$

where

$$
\Phi=-\int_{x}^{0} k(x) d x=\int_{0}^{x} k(x) d x
$$


is a total accumulated phase, and the factor $\exp (-i \omega t)$ is assumed. Since $k(x)=\omega / c(x)=$ $\omega / a x^{n}$, one can see from (2) that the phase $\Phi$ becomes infinite if $n \geq 1$. This means that under these circumstances the wave never reaches the point $x=0$ (the point of singularity). Therefore, it never reflects back either, i.e. the wave becomes trapped, thus indicating that the above mentioned ideal medium with a linear or higher power-law profile of wave velocity can be considered as ‘acoustic black hole’ for the wave under consideration.

This phenomenon has been first described in 1946 by Pekeris [8] for acoustic waves propagating in a stratified ocean characterised by sound velocity profile linearly decreasing to zero with depth. Later on, several other authors have predicted the possibility of the effects of zero reflection for wave phenomena of different physical nature [9-11]. In particular, a practically important possibility of zero reflection of flexural elastic waves from a tip of an ideal quadratic wedge has been predicted by Mironov [11].

\section{B. One-dimensional acoustic black holes for flexural waves}

To understand the phenomenon of acoustic black holes for the case of flexural waves one can consider the simplest one-dimensional case of plane flexural wave propagation in the normal direction towards the edge of a free elastic wedge considered as a plate of variable thickness described by a power-law relationship $h(x)=\varepsilon x^{m}$, where $m$ is a positive number and $\varepsilon$ is a constant (Fig. 1). 


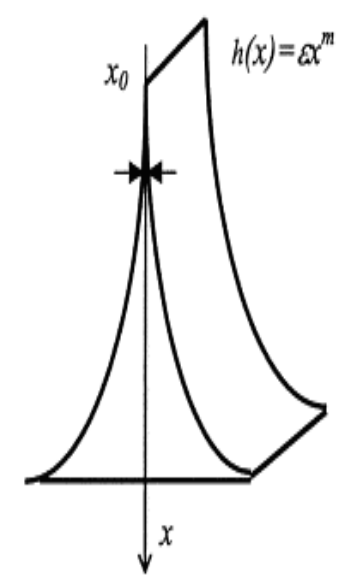

Fig. 1. Geometry of an elastic wedge of power-law profile

Since flexural wave propagation in such wedges can be described in the geometrical acoustics approximation (see [12-15] for more detail), the integrated wave phase $\Phi$ resulting from the wave propagation from an arbitrary point $x$ located in the wedge medium plane to the wedge tip $(x=0)$ can be expressed by (2). In this case $k(x)$ is a local wavenumber of a flexural wave for a wedge in contact with vacuum: $k(x)=12^{1 / 4} k_{p}^{1 / 2}\left(\varepsilon x^{m}\right)^{-1 / 2}$, where $k_{p}=\omega / c_{p}$ is the wavenumber of a symmetrical plate wave (symmetric Lamb mode of the lowest order), $c_{p}=2 c_{t}\left(1-c_{t}^{2} / c_{l}^{2}\right)^{1 / 2}$ is its phase velocity, and $c_{l}$ and $c_{t}$ are longitudinal and shear wave velocities in a wedge material, and $\omega=2 \pi f$ is circular frequency.

Again, one can easily see that the integral in (2) diverges for $m \geq 2$, which corresponds to the earlier discussed condition of linear or faster decrease in phase velocity. This means that the phase $\Phi$ becomes infinite under these conditions, and the wave never reaches the edge. Therefore, it never reflects back either, i.e. the wave becomes trapped, thus indicating that the above mentioned ideal wedges represent acoustic ‘black holes’ for incident flexural waves.

Real fabricated wedges, however, always have truncated edges. And this adversely affects their performance as 'black holes'. If for ideal wedges of power-law shape (with $m \geq 2$ ) it 
follows from (2) that even an infinitely small material attenuation, described by the imaginary part of $k(x)$, would be sufficient for the total wave energy to be absorbed, this is not so for truncated wedges. Indeed, for truncated wedges the lower integration limit in (2) must be changed from 0 to a certain value $x_{0}$ describing the length of truncation. Therefore, for typical (rather low) values of attenuation in such materials as steel, even very small truncations $x_{0}$ result in the reflection coefficients $R_{0}$ becoming as large as $50-70 \%$, which makes it impossible to use such wedges as practical vibration dampers.

It has been proposed by the present author [1-4] that the situation for real wedges (with truncations) can be drastically improved via increasing wave energy dissipation in the area of slow wave velocity (near the sharp edges) by covering wedge surfaces near the edges by thin absorbing layers (films), e.g. by polymeric films. For such systems, one can derive the corresponding analytical expressions for the reflection coefficients of flexural waves from the edges of truncated wedges covered by absorbing layers. For example, for a wedge of quadratic shape, i.e. with $h(x)=\varepsilon x^{2}$, covered by thin absorbing layers on both surfaces the following analytical expression for the resulting reflection coefficient $R_{0}$ can be derived [2, 3]:

$$
R_{0}=\exp \left(-2 \mu_{1}-2 \mu_{2}\right)
$$

where

$$
\begin{gathered}
\mu_{1}=\frac{12^{1 / 4} k_{p}^{1 / 2} \eta}{4 \varepsilon^{1 / 2}} \ln \left(\frac{x}{x_{0}}\right), \\
\mu_{2}=\frac{3 \cdot 12^{1 / 4} k_{p}^{1 / 2} v \delta}{4 \varepsilon^{3 / 2}} \frac{E_{2}}{E_{1}} \frac{1}{x_{0}{ }^{2}}\left(1-\frac{x_{0}^{2}}{x^{2}}\right) .
\end{gathered}
$$


Here $v$ is the loss factor of the material of the absorbing layer and $\delta$ is its thickness, $\eta$ is the loss factor of the wedge material, $x_{0}$ is the wedge truncation length, $E_{1}$ and $E_{2}$ are respectively the Young's moduli of the plate and of the absorbing layer, $x$ is the coordinate of the point of observation taken at a sufficiently large distance from the wedge tip. In the case of a wedge of quadratic shape covered by damping layers on one surface only, equations (3) and (4) remain unchanged, whereas the right-hand side of (5) is multiplied by the factor $1 / 2$.

In deriving equations (3)-(5), the effect of thin absorbing layers on flexural wave velocity has been neglected, which can be done for very thin absorbing layers. The extension of the above analysis to the case of absorbing layers of arbitrary thickness has been carried out in the paper [2].

Note that geometrical acoustics approximation for the above-mentioned quadratic wedges $(m=2)$ is valid for all $x$ provided that the following applicability condition is satisfied [3]:

$$
\frac{12^{1 / 4} k_{p}^{1 / 2}}{\varepsilon^{1 / 2}}>>1 .
$$

For majority of practical situations this condition can be easily satisfied even at relatively low frequencies. For values of power-law exponent $m$ different from 2, the applicability conditions depend on distance $x$. For example, considering the conditions for validity of geometrical acoustics approximation for power-law-profiled wedges with $m=3$ and $m=4$, one can obtain the following applicability conditions for wedges with $m=3$ and $m=4$ respectively [3]:

$$
\begin{gathered}
\frac{3 \varepsilon^{1 / 2} x^{1 / 2}}{2 \cdot 12^{1 / 4} k_{p}^{1 / 2}}<<1, \\
\frac{2 \varepsilon^{1 / 2} x}{12^{1 / 4} k_{p}^{1 / 2}}<<1 .
\end{gathered}
$$


As it follows from equations (7) and (8), for such wedges the geometrical acoustics approximation works well for small $x$ and becomes invalid for large distances $x$ from the wedge edges. Note in this connection that for a wedge of linear profile $(m=1)$ characterised by the wedge angle $\theta$, so that $h(x)=\theta x$, it has been shown earlier $[12,13]$ that the abovementioned geometrical-acoustics approximation is valid under the condition

$$
\frac{k_{p^{X}}}{\theta}>>1
$$

In this case, that, of course, does not represent an acoustic black hole, the geometrical acoustics solution breaks down in the immediate vicinity of the wedge edge (at small values of $\left.k_{p} x\right)$ and/or at large wedge angles $\theta$.

Calculations for quadratic wedges $(m=2)$ according to (3)-(5) show that, if an absorbing layer is present $(\delta \neq 0$ and $v \neq 0$ ), it brings a very substantial reduction of the reflection coefficient, sometimes down to 1-3 \% [2, 3], which constitutes the 'acoustic black hole effect'. Thus, in the presence of the absorbing layer (film) the value of the reflection coefficient is much smaller than for a wedge with the same value of truncation, but without a film. Obviously, it is both the specific geometrical properties of a quadratically-shaped wedge in respect of flexural wave propagation and the effect of thin damping layers that result in such a significant reduction of the reflection coefficient.

According to energy conservation law, all wave energy that is not reflected from the free edge is absorbed via its conversion into heat. Note that almost all absorption of the incident wave energy takes place in the vicinity of the sharp edge of a wedge. The same principle of combination of the power-law geometry with the presence of thin absorbing layers can be applied also to beams of power-law profile [5, 16].

The effect of wedge truncation length $x_{0}$ on the reflection coefficient $R_{0}$ in the above example is shown in Fig. 2. For comparison, the curve corresponding to the wedge not 
covered by absorbing layers is shown as well. One can see that the behaviour of the reflection coefficient $R_{0}$ as a function of $x_{0}$ is strongly influenced by the absorbing layers. In particular, the effect of absorbing layers results in drastic reduction of the reflection coefficient for relatively small values of edge truncation $x_{0}$.

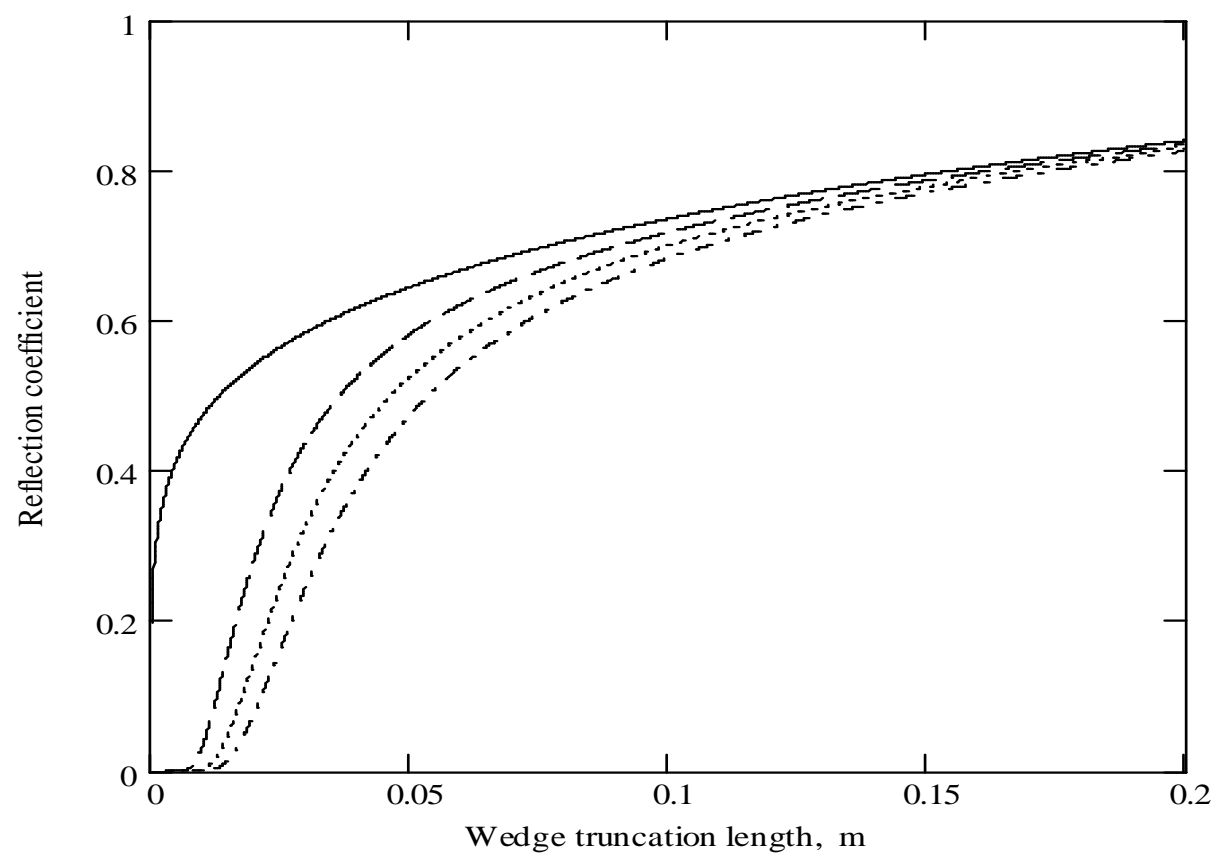

Fig. 2. Effect of wedge truncation length $x_{0}$ on the reflection coefficient of flexural waves $R_{0}$ : solid curve corresponds to an uncovered wedge, dashed, dotted and dash-dotted curves correspond to a wedge covered by absorbing films with the values of $E_{2} / E_{1}$ equal to 0.1 , 0.2 and 0.3 ; the film material loss factor $v$ is 0.15 , and the film thickness $\delta$ is $15 \mu m$ [3]

The discussion so far concerned one-dimensional problems of flexural wave interaction with acoustic black holes, in particular the case of plates of variable thickness for which we analysed only normal incidence of a flexural (bending) wave on a sharp edge. In the case of oblique incidence, one should take into account the non-zero component of the bending wave 
vector in the direction parallel to the edge, which is constant during the whole process of wave propagation. Note, however, that because of the normal component's of the bending wave vector being very large near the edge, the angle of wave approach to the edge is almost normal (see Fig. 3).

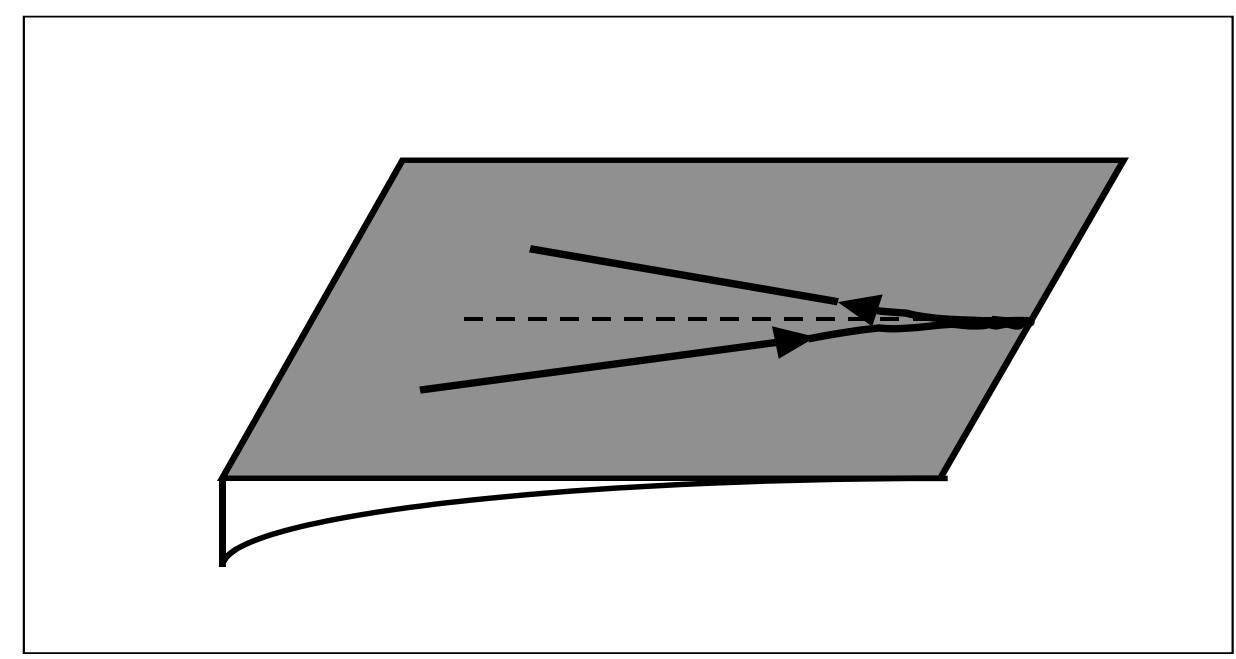

Fig. 3. Reflection of a bending wave from a sharp edge of a quadratic wedge at oblique incidence; both incident and reflected waves are propagating almost in normal direction near the edge.

It is important to recall that the main contribution to the total accumulated phase of the bending wave propagating in a wedge of power-law profile is made at a very small distance near the edge. Therefore, in all practical calculations of the reflection coefficients for bending waves propagating at oblique angles to the edges of wedges of power-law profile it is sufficient to use the above-mentioned one-dimensional results obtained for the case of normal incidence of bending waves. 


\section{Experimental evidence of acoustic black holes for flexural waves}

The first experimental observation of the 'acoustic black hole effect' for a wedge of quadratic profile has been described in the paper [4]. The system under investigation consisted of a steel wedge of quadratic shape covered on one side by a strip of absorbing layer located at the sharp edge. The wedge dimensions were: $280 \mathrm{~mm}$ (length) and $200 \mathrm{~mm}$ (width). Its thickness at the thick end was $4.5 \mathrm{~mm}$, and the value of the quadratic wedge parameter $\varepsilon$ was $5 \times 10^{-5} \mathrm{~mm}^{-1}$. The mass density of the wedge material (steel) was $7900 \mathrm{~kg} / \mathrm{m}^{3}$, and the velocities of longitudinal and shear elastic waves were equal to $5900 \mathrm{~m} / \mathrm{s}$ and $3200 \mathrm{~m} / \mathrm{s}$ respectively. The mass density of the absorbing layer made of usual adhesive electro-isolating tape was $1160 \mathrm{~kg} / \mathrm{m}^{3}$, with the velocity of longitudinal waves equal to $1900 \mathrm{~m} / \mathrm{s}$. Measurements of point mobility, i.e. particle vibration velocity related to the force applied at the same location on the wedge (also known as driving point admittance), have been carried out in the frequency range 100-6500 Hz. Measurements have been made for a free wedge, for a wedge covered by narrow strips of thin absorbing layers (e.g. by the above-mentioned isolating tapes), and for a free and covered reference plates of constant thickness $h=4.5 \mathrm{~mm}$ having the same length and width as the above-mentioned quadratic wedge.

The results of the measurements of point mobility are shown in Fig. 4. It can be seen that, in agreement with the theory, due to a strong suppression of flexural wave reflections from one side of the wedge (the sharp edge), a very significant reduction of resonant peaks (up to $20 \mathrm{~dB}$ ) can be observed in a wedge covered by an absorbing layer, in comparison with the uncovered wedge or with the reference plates of constant thickness. This demonstrates that vibration damping systems utilising the acoustic black hole effect are efficient and suitable for practical applications. 


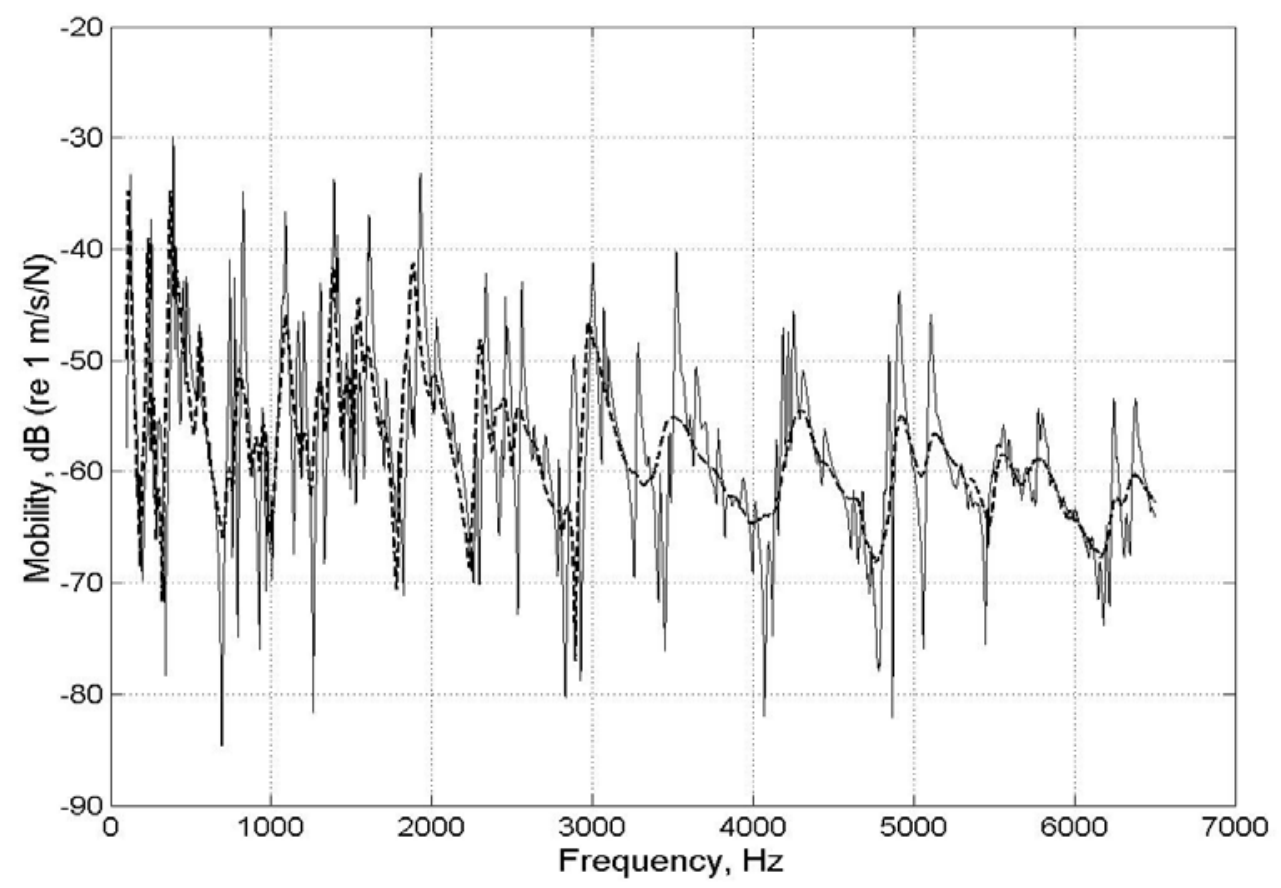

Fig. 4. Measured point mobility of a quadratic steel wedge: free wedge (solid curve) and wedge covered by a thin adhesive strip of thickness $\delta=0.2 \mathrm{~mm}$ (dashed curve) [4]

The main advantage of vibration damping devices using the acoustic black hole effect in comparison with traditional methods of damping that use covering entire surfaces of damped structures by rather thick layers of absorbing materials is that the amount of absorbing materials required for systems using the acoustic black hole effect is very small. This can be attractive for design engineers and developers who may prefer to use such systems in cases where using traditional methods of vibration damping results in unacceptable increase in mass of the structure, for example in some aeronautical and automotive applications. 


\section{Two-dimensional acoustic black holes for flexural waves}

Two-dimensional acoustic black holes for flexural waves, such as protruding cylindrically symmetrical indentations (pits) drilled in a regular thin plate of constant thickness (see Fig. 5), have been first proposed and investigated by the present author [5] using geometrical acoustics approach.

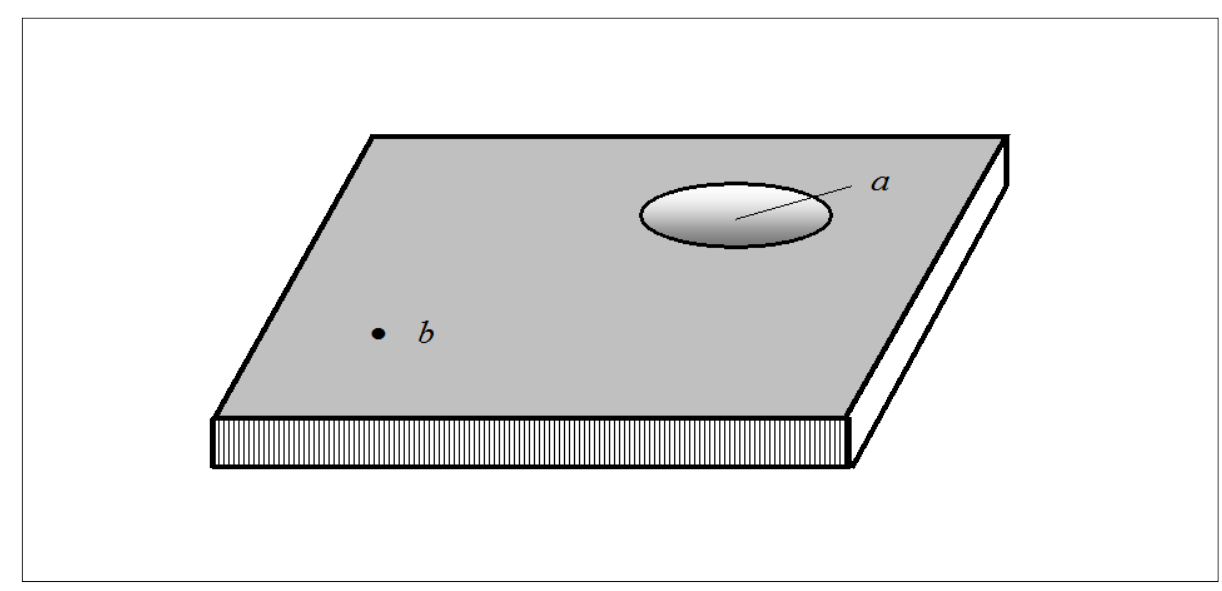

Fig. 5. A protruding cylindrically symmetrical pit of power-law profile materialising a twodimensional acoustic black hole for flexural waves [5]

To consider flexural (bending) wave propagation over two-dimensional pits of power-law profile it is convenient to use a geometrical acoustics approach in Hamiltonian formulation similar to that earlier applied by the present author [17] to analysing Rayleigh surface wave propagation across smooth large-scale surface irregularities (see also the monograph [14]). Application of this approach to the case of flexural wave propagation in thin plates of variable thickness, including plates with cylindrically symmetrical pits, is even simpler than 
in the case of Rayleigh waves on curved surfaces as in the former case there is no geometrically induced anisotropy of flexural wave velocity.

The equations defining ray trajectories for the case of flexural wave propagation over cylindrically symmetrical pits can be written in the form [5]:

$$
\begin{aligned}
& \frac{d r}{d \theta}=r \frac{1}{\tan \alpha}, \\
& \frac{d \alpha}{d \theta}=-1-\frac{r}{n} \frac{\partial n}{\partial r}, \\
& n r \sin \alpha=\text { const. }
\end{aligned}
$$

Here $r$ and $\theta$ are polar coordinates associated with the centre of the pit and measured along the plate's middle plane, $\alpha$ is the angle between the vectors $\boldsymbol{r}$ and $\boldsymbol{k}$, where $\boldsymbol{k}$ is the wave vector of the flexural wave, $n(r)=c_{0} / c(r)$ is the index of refraction of the flexural wave in the pit area, and $c_{0}$ and $c=c(r)$ are the flexural wave phase velocities in the basic plate of constant thickness $h_{0}$ (away from the pit) and in the pit area respectively. Obviously, $n(r)=$ $h_{0}^{1 / 2} / h(r)^{1 / 2}$, where $h(r)$ is the local thickness of a plate in the pit area. In what follows we assume that, like in the above-mentioned case of one-dimensional wedges, $h(r)$ is described by a power-law relationship, i.e. $h(r)=\varepsilon r^{m}$.

Note that the last equation in (10) represents Snell's law in the axisymmetrical case. It is convenient to rewrite this equation in the form linking the initial point of the ray trajectory outside the pit (in particular, this can be an excitation point), characterised by the values $r_{0}$, $\alpha_{0}$ and $n_{0}=1$, with the point of observation in the pit area, characterised by the values $r, \alpha$ and $n$ : 


$$
n r \sin \alpha=r_{0} \sin \alpha_{0}=\rho
$$

Here we have introduced the so-called impact parameter $\rho=r_{0} \sin \alpha_{0}$, which is important for determination of ray trajectories for different values of $\alpha_{0}$. For the given values of $r_{0}$, and $\alpha_{0}$, this parameter describes the closest distance between the ray trajectory and the centre of the polar coordinate system in the absence of a pit (in this case the trajectories are straight lines). The presence of a pit influences the rays that propagate through it, and the corresponding ray trajectories are no longer straight lines.

The analysis shows that, in the case of symmetrical pits of power-law profile with $m \geq 2$, a number of rays that are close enough to a direct ray, including a direct ray itself, deflect towards the centre of the pit (see Fig. 6).

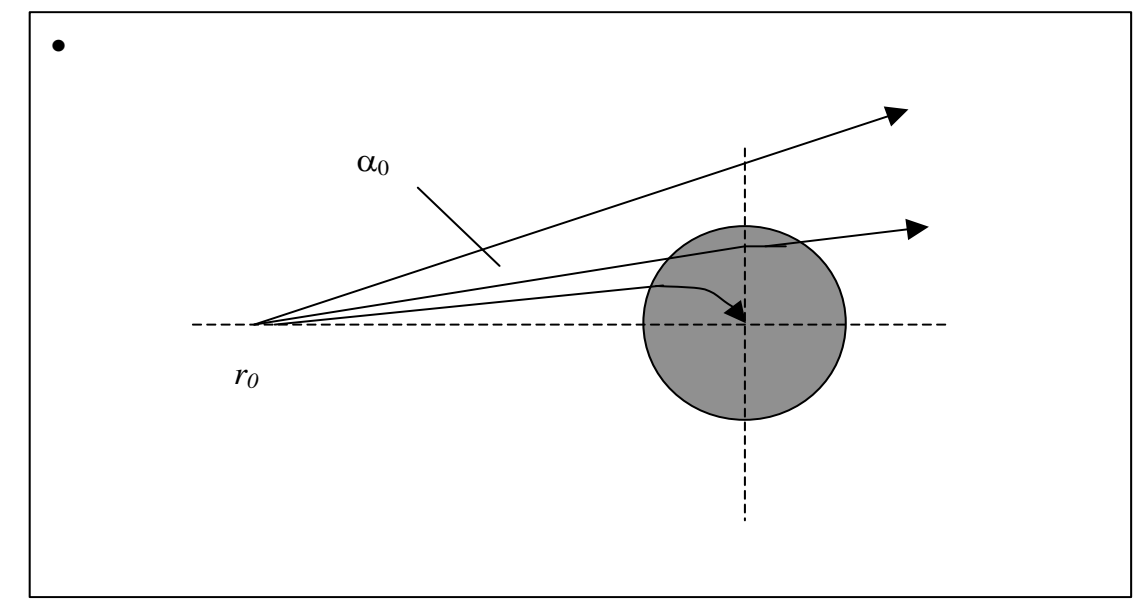

Fig. 6. Typical ray trajectories illustrating propagation of flexural waves over a pit of powerlaw profile; the ray below is trapped by the acoustic black hole [5]

Since the central (nearly protruding) area of the pit (after covering by a small amount of absorbing material) makes a major contribution to the reflection coefficients, one can use one- 
dimensional calculations for the reflection coefficients of all such rays, like in the abovementioned case of oblique incidence of a bending wave on the edge of a wedge (see Fig. 3). Since the central area of the pit covered by absorbing material (not shown in Figs. 5 and 6) acts as an efficient absorber for flexural waves, all such 'captured' rays can be considered as fully absorbed rays that have taken away part of the energy of the incident wave. This results in damping of resonant vibrations of finite structures.

From the practical point of view, the above-mentioned two-dimensional acoustic black holes (indentations of power-law profile) can be placed at any point of a plate or any other plate-like or shell-like structure. The effect of such a black hole will be in eliminating some rays, intersecting with the black hole, from contributing to the overall frequency response function of a structure, which will result in substantial damping of resonant vibrations. To amplify the vibration damping effect of two-dimensional acoustic black holes one can place ensembles of several black holes distributed over the structure (e.g. periodic arrays of black holes) [5], if this does not compromise its main functions, e.g. its rigidity. First experimental investigations of two-dimensional acoustic black holes in different types of plates have been carried out in [18-20].

As was briefly mentioned above, one of the most important advantages of such onedimensional and two-dimensional acoustic black holes as dampers of structural vibrations is that they are efficient even for relatively thin and narrow strips of attached absorbing layers. The reason for this is that wave energy dissipation takes place mainly in a very narrow area near sharp edges. This is in contrast with the traditional techniques employing covering the whole surfaces of structures by relatively thick layers of absorbing materials [21, 22]. And this important feature of acoustic black holes can be very attractive for many practical applications, especially for those involving light-weight structures. 


\section{E. Optic black holes and their comparison with acoustic black holes}

Recently, the above-mentioned basic principle of power-law reduction in velocity accompanied by efficient energy absorption in the 'slow' area has been re-invented in optics to create the so-called 'optic black holes' [6, 7]. In particular, it has been shown in [6] that a spherical or a cylindrical layer with dielectric constant $\varepsilon=\varepsilon(r)$ altering with distance $r$ according to the inverse power law: $\varepsilon \sim 1 / r^{n}$, with $n \geq 2$, can capture incident light and direct it towards the absorbing core. Since the velocity of light is inversely proportional to $(\varepsilon)^{1 / 2}$, the condition $\varepsilon \sim 1 / r^{n}$, where $n \geq 2$, implies that the velocity of light should experience linear or higher order power-law type decrease towards the centre. This is exactly the same type of velocity reduction that has been considered above for the general case of wave propagation, regardless of the physical nature of the wave. The above-mentioned optic black holes have attracted attention of the optics research community and practitioners as effective means of harvesting solar energy and its conversion into heat.

Note that the method of analysis used in [6], described by the authors as 'semiclassical approach' and 'Hamiltonian mechanics', is in fact equivalent to the geometrical acoustics (optics) approach in Hamiltonian formulation used in [5] for the analysis of two-dimensional acoustic black holes for flexural waves. Therefore, it is quite obvious that the principles and theories of the above-mentioned acoustic and optic black holes are almost identical (see also [23]). Apparently, the authors of $[6,7]$ were unaware of the above-mentioned earlier papers on acoustic black holes [1-5], let alone the papers on zero reflection properties of media with wave velocities varying with distance according to power-law profiles [8-11]. 


\section{Some recent investigations of the acoustic black hole effect}

\section{A. Rectangular plates with attached wedges of power-law profile}

One of the types of structures that have been recently investigated at Loughborough University both theoretically and experimentally included plates or beams bounded by the attached power-law wedges, with the addition of small amounts of absorbing material at their sharp edges $[24,25]$. The above-mentioned wedges-like structures (see Fig. 7) materialise one-dimensional acoustic black holes for flexural waves placed at the edges of basic rectangular plates vibrations of which are to be damped. The experiments have demonstrated that power-law wedges covered by narrow strips of thin absorbing layers, when attached to edges of rectangular plates, are much more efficient dampers of structural vibrations than traditional rather thick layers of absorbing materials covering entire plate surfaces.

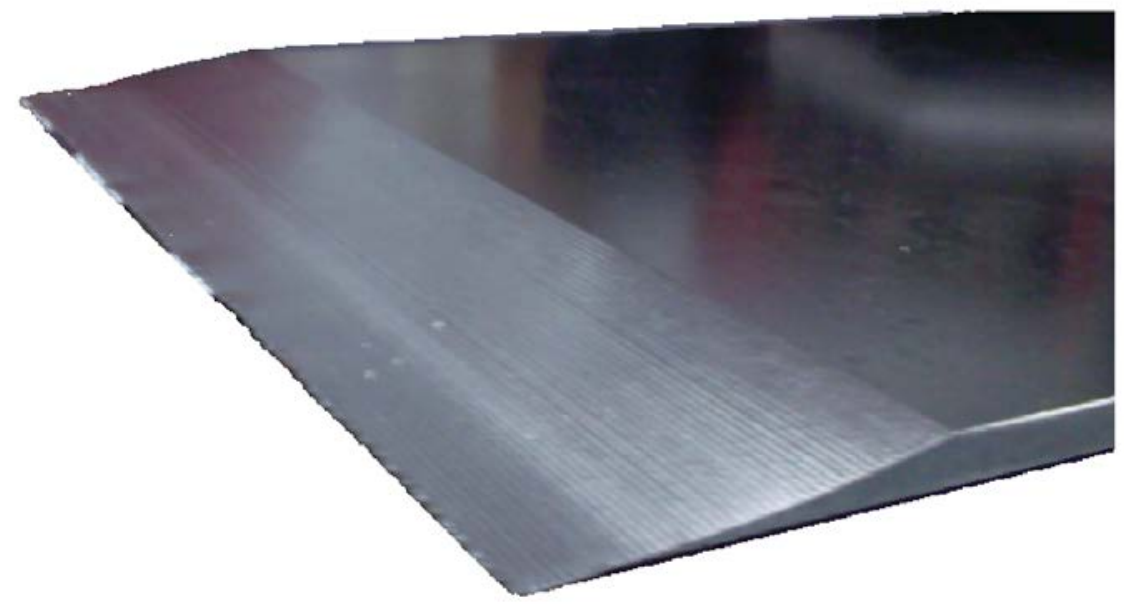

Fig. 7. Photo showing part of a rectangular plate bounded by a power-law profiled wedge (with $m=2.2$ ) [24] 
In the paper [25], the effects of deviations of real manufactured wedge-like structures from ideal elastic wedges of power-law profile on damping flexural vibrations have been investigated experimentally. Namely, the effect of mechanical damage to wedge tips has been studied, including tip curling and early truncation, as well as the placement of absorbing layers on different wedge surfaces. Also, the effects of welded and glued bonding of wedge attachments to basic rectangular plates (strips) have been studied. In particular, it has been found that a tip damage (curling), resulting in a wedge with an extended sharp edge, is not detrimental for its damping performance. On the contrary, the extended wedge provided a very efficient damping. Note that similar experimental results have been obtained independently by Bayod [26] who investigated vibration damping in a power-law wedge extended at the sharp edge to form a thin plate of constant thickness, which was made specifically to overcome difficulties associated with manufacturing of very sharp wedges. It also has been demonstrated in [25] that attaching power-low profiled wedges to a rectangular plate (strip) by welding or via glue results in damping performance that generally is not worse than the performance of homogeneous samples containing the same wedges at their edges. The main and important conclusion that can be drawn from these investigations is as follows. Although the above-mentioned geometrical and material imperfections generally reduce the damping efficiency to various degrees, the method of damping utilising the acoustic black hole effect is robust enough and can be used widely without the need of high precision manufacturing.

\section{B. Plates containing two-dimensional acoustic black holes}

Other important structures that have been investigated at Loughborough were rectangular plates with tapered indentations (pits) of power-law profile drilled inside the plates [27-30]. In 
the case of quadratic or higher-order profiles, such pits materialise two-dimensional acoustic black holes for flexural waves. Two-dimensional black holes and their ensembles offer an important advantage in comparison with the case of one-dimensional acoustic black holes (wedges of power-law profile). Namely, the potentially dangerous sharp edges of power-law wedges can be eliminated.

Theoretical and experimental investigations have been carried out for a specific case of circular indentations of power-law profile drilled in the centre of a circular plate $[27,28]$. The advantage of this geometrical configuration is that it facilitates theoretical calculations of the frequency response functions. Such calculations have been carried out, and they have shown good agreement with the experimental measurements.

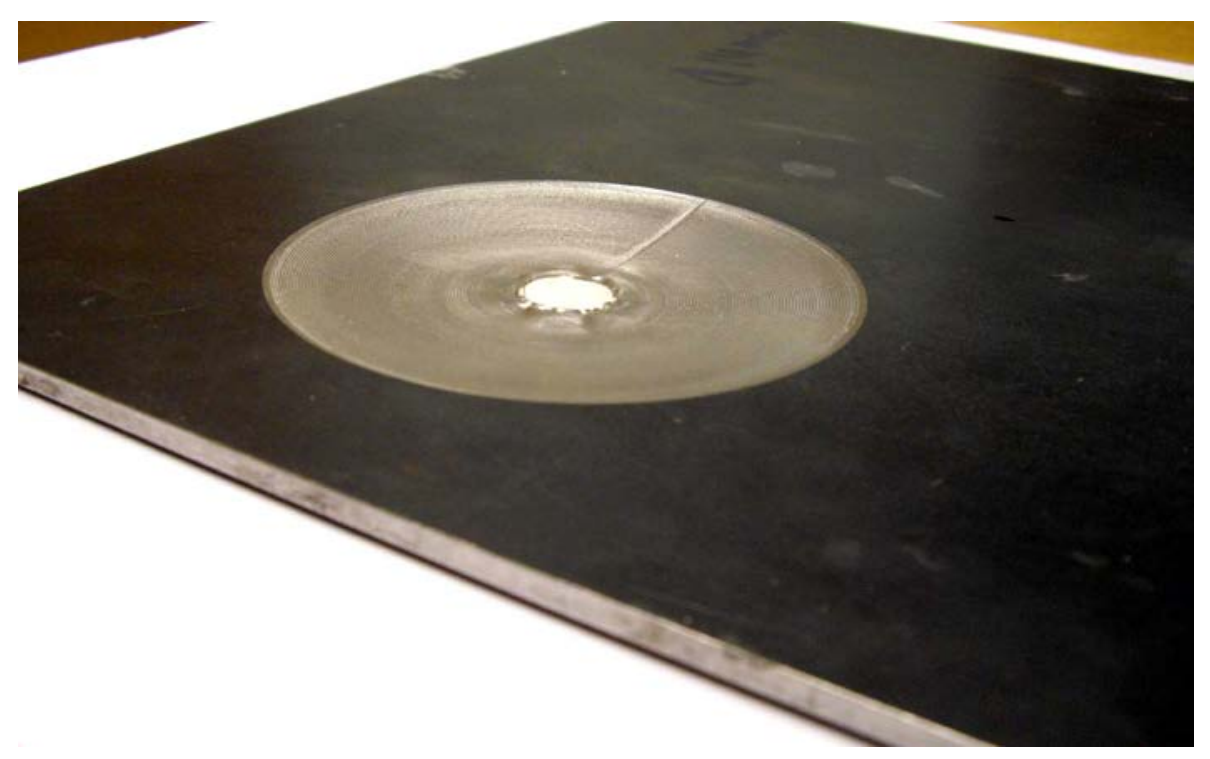

Fig. 8. Circular indentation of power-law profile materialising a two-dimensional acoustic black hole for flexural waves in plates. 
In the paper [29], it has been demonstrated that basic power-law indentations that are just protruding over the opposite plate surface (Fig. 5) cause very small reduction in resonant peak amplitudes, which may be due to their relatively small absorption cross-section. Introduction of a $2 \mathrm{~mm}$ central hole improved the situation and increased damping. To increase damping even more, the absorption cross-section has been enlarged by increasing the size of the central hole in the indentation up to $14 \mathrm{~mm}$, while keeping the edges sharp (see Fig. 8). As expected, such pits, becoming in fact curved power-law wedges, resulted in substantially increased damping that was comparable with that achieved by one-dimensional wedges of power-law profile.

To make such two-dimensional acoustic black holes even more efficient, ensembles of several (up to six) black holes have been manufactured (Fig. 9) and investigated experimentally [29, 30].

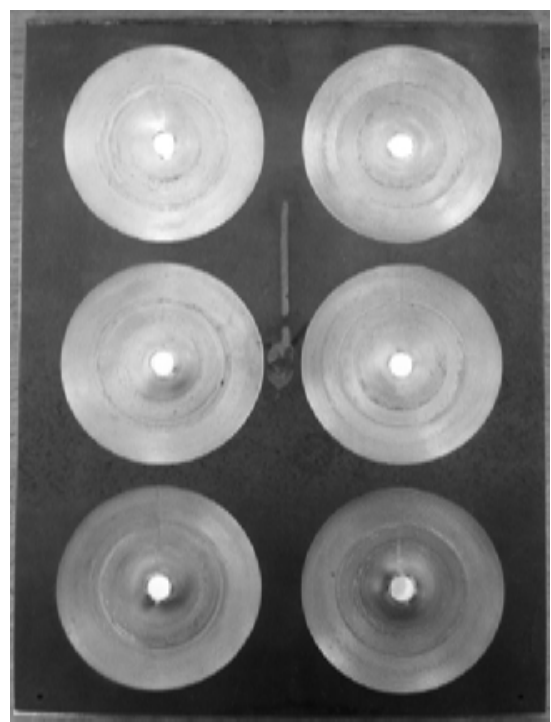

Fig. 9. Manufactured steel plate containing an array of six two-dimensional acoustic black holes [29, 30] 
Figure 10 shows the measured frequency response for a rectangular plate shown in Fig. 9 and containing six power-law profiled circular indentations with central holes and with added pieces of damping layers, in comparison with the response of a reference plate of the same size [29]. As it can be seen, the damping at resonant peaks caused by acoustic black holes is quite substantial, up to $15 \mathrm{~dB}$ at higher frequencies.

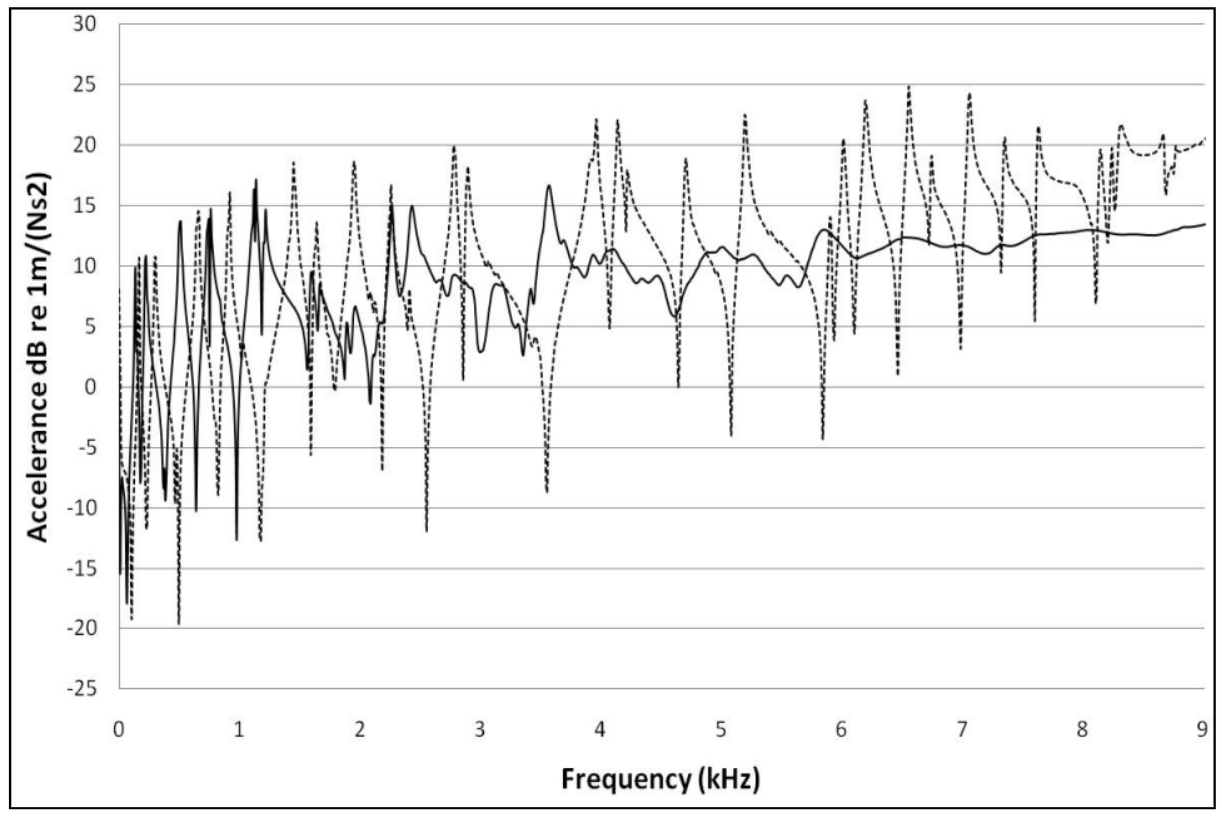

Fig. 10. Measured accelerance for a plate containing six profiled circular indentations with $14 \mathrm{~mm}$ central holes and additional damping layers (solid line), as compared to a reference plate (dashed line) [29]

In paper [30], the sound power radiated by a rectangular plate with six indentations shown in Fig. 9 has been measured and compared with the radiation of a reference plate of constant thickness having the same geometrical dimensions. It has been shown that acoustic black 
holes, as expected, cause a significant reduction in radiated sound power, in spite of some local increase in plate vibration levels at the black holes themselves.

\section{Some other acoustic black hole configurations and applications}

Among other promising types of acoustic black-hole geometry that have been recently studied at Loughborough were slots of power-law profile made inside rectangular plates [31]. As was mentioned above, one of the problems faced by one-dimensional black holes formed by power-law wedges attached to edges of structures is having sharp wedge tips exposed on the outer edges. One of the solutions to this problem is the two-dimensional black holes described in the previous section. Slots of power-law profile placed inside structures represent another possible solution that moves the power-law wedges inside a plate, so that they form edges of power-law slots within the plate (Fig. 11).

Different configurations of such slots in steel and composite plates and panels have been manufactured and tested experimentally. It has been demonstrated that slots of power-law profile located within plates and panels materialise a specific type of quasi-one-dimensional acoustic black holes for flexural waves and represent an effective method of damping structural vibrations. The maximum damping achieved on a steel plate with a slot was about $11 \mathrm{~dB}$. 


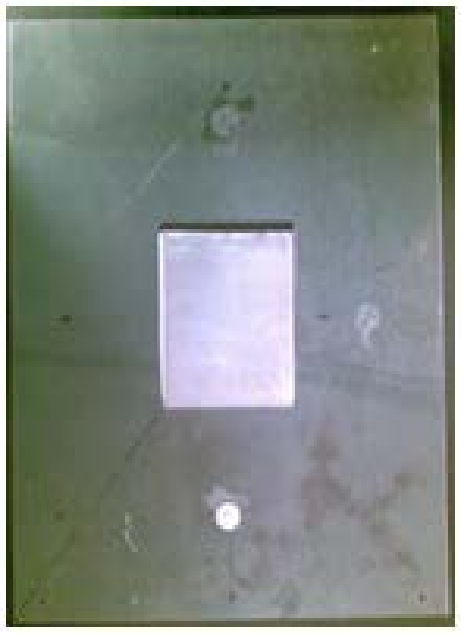

(a)

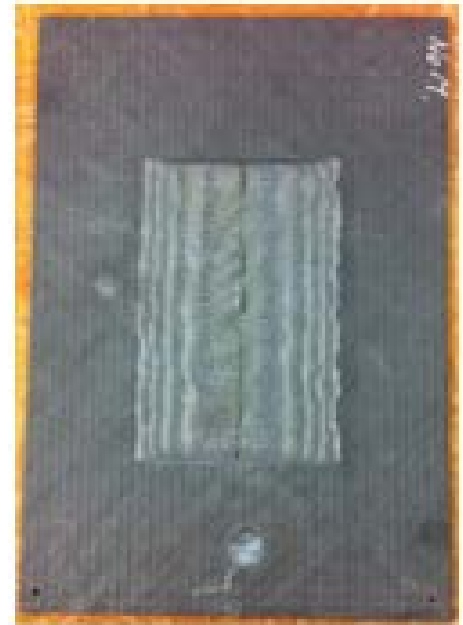

(b)

Fig. 11. Steel (a) and composite (b) plates containing slots of power-law profile [27]

Following the idea first proposed in [4], experimental investigations into damping of flexural vibrations in turbofan blades with trailing edges tapered according to a power-law profile have been carried out in the papers [32, 33]. Obviously, wedges of power-law profile fit naturally with a fan blade geometry (see Fig. 12). Several aluminium samples of model fan blades have been manufactured, including straight and twisted samples, the latter being more realistic models for real fan blades. All model blades were excited by an electromagnetic shaker, and the associated frequency response functions have been measured. The measurements have demonstrated that power-law tapering of trailing edges of turbofan blades results in substantial damping of resonant peaks even in the case of twisted samples. Thus, the above-mentioned power-law tapering of trailing edges of fan blades can be considered as a viable method of reduction of blade vibrations. 


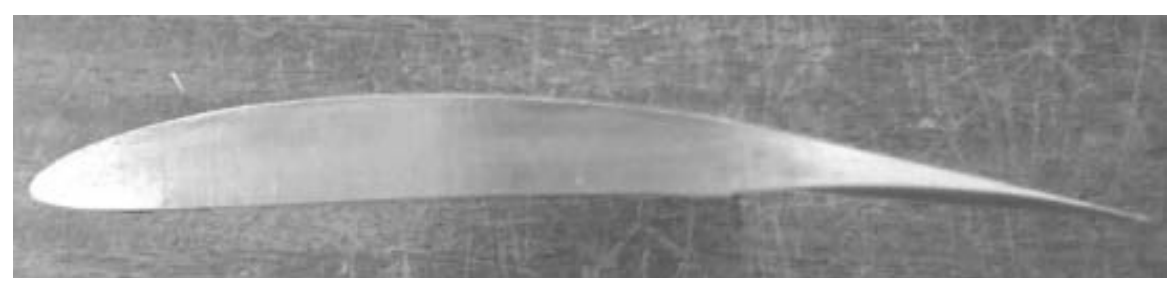

Fig. 12. Profile of a model fan blade with a trailing edge tapered to form a wedge of powerlaw shape [32]

In the papers [34, 35], experimental investigations of one- and two-dimensional acoustic black holes (power-law wedges and circular indentations) made in composite plates and panels (Fig. 13) have been carried out. The addition of acoustic black holes to composite plates and panels resulted in further increase in damping of resonant vibrations, in addition to the already substantial inherent damping due to large values of the loss factor for composites $(0.1-0.2)$. Note that, due to large values of the loss factor for composite materials used, no additional layers of absorbing material were required, as expected.

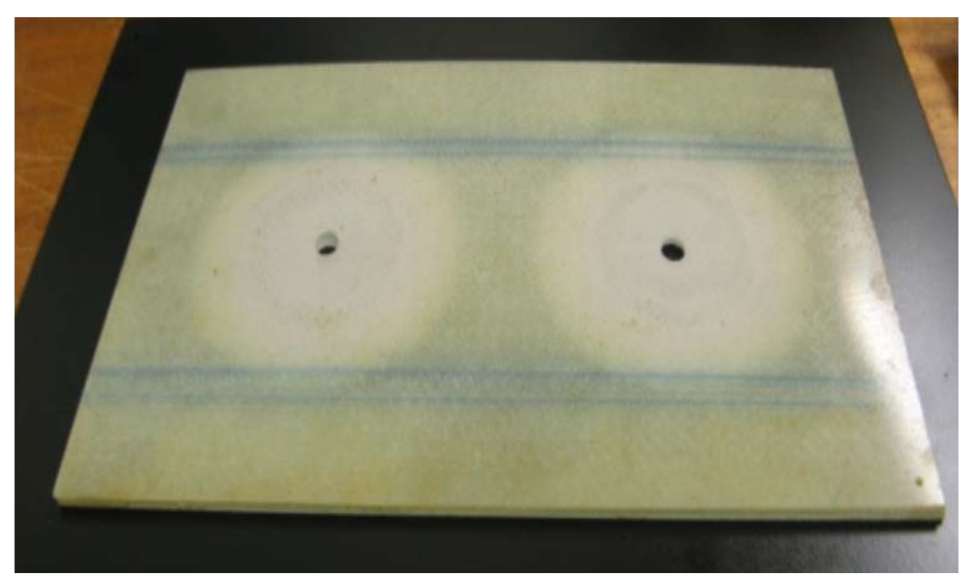

Fig. 13. Glass fibre composite plate with two two-dimensional acoustic black holes [34] 
The results of these and earlier mentioned experimental investigations have demonstrated that in all of the above-mentioned cases the efficiency of vibration damping based on the acoustic black hole effect is substantially higher than that achieved by traditional methods. The key advantage of using the acoustic black hole effect for damping structural vibrations is that it requires very small amounts of added damping materials, which is especially important for damping vibrations in light-weight structures used in aeronautical and automotive applications.

\section{Acoustic black holes for sound absorption in gases and liquids}

It would be natural to extend the above-mentioned successful practical implementations of acoustic black holes for flexural waves to the case of acoustic black holes designed for sound absorption in gases and liquids. Such black holes could be used for sound absorption and noise control both in open areas and inside enclosed spaces.

There are still very few investigations in this area. In the first theoretical paper on acoustic black holes for sound absorption in air [36], it was proposed to use an inhomogeneous acoustic waveguide with walls of variable impedance materialised via quasi-periodic ribbed structure to achieve the required linear decrease in acoustic wave velocity with propagation distance. No absorbing core was considered in this work. Therefore, to make this structure work in real situations one should consider adding sound absorbing materials in the area of low sound velocity.

Another possibility of creating acoustic black holes for absorption of sound in air is to use specially designed graded metamaterials as wave retarding structures, as it was done in the case of optic black holes $[6,7]$. The first consideration of this possibility has been made in the 
paper [37] using graded sonic crystals formed by circular arrays of small scatterers with their filling fractions varying along the radius. In an array of small rigid circular cylinders, the effective density varies with filling fraction. This means that by varying the filling fraction the desired dependence of the effective density, and hence the required dependence of sound velocity on $r$, can be achieved. This method of wave velocity reduction has been implemented, and an absorbing core (a porous material) has been added to form the acoustic black hole [37].

The above-mentioned two types of acoustic black holes for sound absorption in air require further investigations, both theoretical and experimental. The expected outcome is to develop efficient sound absorption devices that would require much smaller amounts of absorbing material and thus would be lighter and more convenient than the existing sound absorbing panels.

In contrast to the cases of acoustic black holes for flexural waves and optic black holes, there is a serious problem associated with the development of acoustic black holes for sound absorption in air. This is the fact that at typical acoustic frequencies, the acoustic wavelengths are comparable with expected geometrical dimensions of practical black holes, which can make them non-operational at low and medium frequencies. The use of quicker varying power-law profiles could help to make black holes operational at medium frequencies.

It also seems beneficial to consider possible new types of geometry of acoustic black holes, in addition to circular cylinders and spheres [37]. In particular, similarly to elastic wedges of power-law profile implementing one-dimensional black holes for flexural waves, one can propose quasi-one-dimensional (flat) acoustic black holes for sound absorption in air (with the absorbing core placed on one side). Such devices could be attached to walls of an enclosure to reduce the overall noise level. Also, following the geometry of power-law slots within plates representing quasi-one-dimensional double-sided black holes for flexural waves 
[31], one can propose double-sided quasi-one-dimensional acoustic black holes (with the absorbing core placed in the middle). Such black holes could be suspended on cables to absorb noise on both sides. To amplify the effect of acoustic black holes in air, ensembles or periodic arrays of black holes can be used.

In addition to the above-mentioned two types of acoustic black holes for sound absorption in air, we consider here a new interesting possibility of creating acoustic black holes for absorption of sound propagating in liquid-filled flexible pipes with walls of variable thickness (Fig. 14).

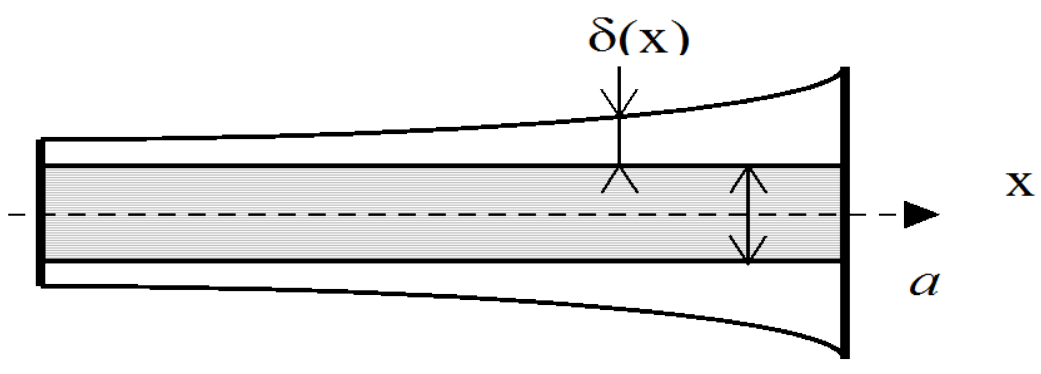

Fig. 14. Geometry of a liquid-filled pipe with flexible walls of variable thickness

Let us start with the well-known Korteweg-Lamb expression for the velocity $c$ of the lowest order acoustic mode propagating in a flexible pipe (see e.g. [38, 39]):

$$
c=c_{0}\left[1+\frac{2 a\left(1-v^{2}\right)}{\delta E \beta_{0}}\right]^{-1 / 2} .
$$


Here $c_{0}$ is the sound velocity in open liquid, $\beta_{0}$ is the compressibility of the liquid, $a$ is the internal radius of the pipe, $\delta$ is the thickness of its flexible walls, $E$ and $v$ are the Young's modulus and the Poisson's ratio of the pipe material respectively (see Fig. 14).

It can be seen from (12) that, e.g. for a rubber pipe filled with water, the second term in square brackets can be much larger than one. If we consider this case and assume that the local thickness of the pipe walls $\delta$ depends on propagation distance $x$ as $\delta=\varepsilon x^{m}$, where $m$ is a positive number and $\varepsilon$ is a constant, then the approximate expression for $c=c(x)$ will take the form:

$$
c(x)=\left[\frac{\varepsilon E}{2 a \rho_{0}\left(1-v^{2}\right)}\right]^{1 / 2} x^{m / 2} .
$$

Substituting the wavenumber $k(x)=\omega / c(x)$ into (2), where $c(x)$ is defined by (13), one can see that the total accumulated phase $\Phi$ becomes infinite if $m \geq 2$, i.e. when the velocity $c$ goes to zero at $x \rightarrow 0$ linearly or faster, which is the necessary condition of the acoustic black hole effect. Adding an absorbing core in the area of low wave velocity, e.g. a piece of porous material, would make this system work in real truncated pipes.

Exciting new opportunities occur if a liquid in the pipe with flexible walls of power-law thickness flows with a non-zero velocity $v$, e.g. in the case of a hose pipe. Then for all $x$ below a certain distance $x_{n r}$ there would be $c(x)<v$. This would make $x_{n r}$ a point of no return for the incident sound, thus materialising an acoustic black hole with a non-zero event horizon. Note in this connection that, in contrast to some works concerned with the development of acoustic analogues of astrophysical (gravitational) black holes using supersonic fluid flows, including a phononic version of Hawking radiation (see e.g. [40-43]), the main aim of the investigations described in the present paper remains finding more 
efficient ways of vibration damping and sound absorption. This author believes that acoustic black holes represent one of the most elegant and smart solutions to this problem.

\section{Conclusions}

The results of the recent theoretical and experimental investigations demonstrate that the efficiency of vibration damping based on the acoustic black hole effect is substantially higher than that achieved by traditional methods.

The key advantage of using the acoustic black hole effect for damping structural vibrations is that it requires very small amounts of added damping materials, which is especially important for damping vibrations in light-weight structures used in aeronautical and automotive applications.

The main disadvantage of this method of damping is the requirement to introduce powerlaw wedges or indentations into structures to be damped, which may compromise their rigidity or integrity. Therefore, this method is limited to some specific structures and applications where structural rigidity and integrity are either not so important or suitable for improvement.

Research into acoustic black holes for sound absorption is still in its infancy. Further investigations are needed to explore their full potential and test their viability. 


\section{References}

[1] V.V. Krylov, "Laminated plates of variable thickness as effective absorbers for flexural vibrations", Proceedings of the 17th International Congress on Acoustics, Rome, Italy, 2-7 September 2001 (Ed. A. Alippi), vol. 1, 2001, pp. 270-271.

[2] V.V. Krylov, "New type of vibration dampers utilising the effect of acoustic 'black holes’", Acta Acustica united with Acustica, vol. 90(5), pp. 830-837, 2004.

[3] V.V. Krylov and F.J.B.S. Tilman, "Acoustic 'black holes' for flexural waves as effective vibration dampers", Journal of Sound and Vibration, vol. 274, pp. 605-619, 2004.

[4] V.V. Krylov and R.E.T.B. Winward, "Experimental investigation of the acoustic black hole effect for flexural waves in tapered plates", Journal of Sound and Vibration, vol. 300, pp. 43-39, 2007.

[5] V.V. Krylov, "Propagation of plate bending waves in the vicinity of one- and twodimensional acoustic 'black holes'", Proceedings of the ECCOMAS International Conference on Computational Methods in Structural Dynamics and Earthquake Engineering (COMPDYN 2007), Rethymno, Crete, Greece, 13-16 June 2007, [CD-ROM].

[6] E.E. Narimanov and A.V. Kildishev, "Optical black hole: Broadband omnidirectional light absorber", Applied Physics Letters, vol. 95, pp. 041106-3, 2009.

[7] A.V. Kildishev, L.J. Prokopeva and E.E. Narimanov, "Cylinder light concentrator and absorber: theoretical description", Optics Express, vol. 18(16), pp. 16646-16662, 2010.

[8] C.L. Pekeris, "Theory of propagation of sound in a half-space of variable sound velocity under condition of formation of a shadow zone", J. Acoust. Soc. Amer., vol. 18, pp. 295315, 1946. 
[9] S.I. Badulin, L.S. Tsimring and V.I. Shrira, "Trapping and vertical focusing of internal waves in the pycnocline due to horizontal inhomogeneities of the stratification and currents", Dokl. Akad. Nauk SSSR, vol. 273(2), pp. 459-463, 1983.

[10] L.D. Landau and E.M. Lifshits, Quantum Mechanics: Non-Relativistic Theory, Pergamon Press, Oxford-New York, 1977.

[11] M.A. Mironov, "Propagation of a flexural wave in a plate whose thickness decreases smoothly to zero in a finite interval", Soviet Physics - Acoustics, vol. 34, pp. 318-319, 1988.

[12] V.V. Krylov, "Conditions for validity of the geometrical-acoustics approximation in application to waves in an acute-angle solid wedge", Soviet Physics -Acoustics, vol. 35(2), pp. 176-180, 1989.

[13] V.V. Krylov, "Geometrical-acoustics approach to the description of localized vibrational modes of an elastic solid wedge", Soviet Physics - Technical Physics, vol. 35(1), pp. 137-140, 1990.

[14] S.V. Biryukov, Yu.V. Gulyaev, V.V. Krylov and V.P. Plessky, Surface Acoustic Waves in Inhomogeneous Media, Springer, Berlin, Heidelberg, 1995, Chapter 9.

[15] V.V. Krylov, "Geometrical acoustics approximation for Rayleigh and Lamb waves", Proceedings of the International Conference on Conditional Monitoring (CM 2012), London, 11-14 June, 2012, [CD-ROM].

[16] V. Kralovic and V.V. Krylov, "Damping of flexural vibrations in tapered rods of power-law profile: Experimental studies", Proceedings of the Institute of Acoustics, vol. 29(5), pp. 66-73, 2007.

[17] V.V. Krylov, "Transmission of Rayleigh waves through smooth large-scale surface irregularities", Soviet Physics - Acoustics, vol. 34(6), pp. 613-618, 1988. 
[18] V. Georgiev, J. Cuenca, M.A. Molerón-Bermúdez, F. Gautier, L. Simon and V.V. Krylov, "Numerical and experimental investigation of the acoustic black hole effect for vibration damping in beams and elliptical plates", Proceedings of the International Conference “Euronoise 2009”, Edinburgh, UK, 26-28 October 2009, [CD-ROM].

[19] E.P. Bowyer, D.J. O’Boy, V.V. Krylov and F. Gautier, "Experimental investigation of damping flexural vibrations using two-dimensional acoustic 'black holes’", Proceedings of the International Conference on Noise and Vibration Engineering (ISMA 2010), Leuven, Belgium, 20-22 September 2010 (Ed. P. Sas, B. Bergen), 2010, pp. 1181-1192.

[20] V.B. Georgiev, J. Cuenca, F. Gautier, L. Simon and V.V. Krylov, "Damping of structural vibrations in beams and elliptical plates using the acoustic black hole effect", Journal of Sound and Vibration, vol. 330, pp. 2497-2508, 2011.

[21] M. Heckl, L. Cremer and E. Ungar, Structure Borne Sound, 2nd Edition, Springer, Berlin, Heidelberg, 1988.

[22] D.J. Mead, Passive Vibration Control, Wiley, Chichester, 1998.

[23] V.V. Krylov, "Acoustic black holes and their applications for vibration damping and sound absorption", Proceedings of the International conference on Noise and Vibration Engineering (ISMA 2012), Leuven, Belgium, 17-19 September 2012 (Sas, P., Moens, D., Jonckheere, S. (ed)), pp. 933-944.

[24] D.J. O’Boy, V.V. Krylov and V. Kralovic, "Damping of flexural vibrations in rectangular plates using the acoustic black hole effect", Journal of Sound and Vibration, vol. 329, pp. 4672-4688, 2010.

[25] E.P. Bowyer, D.J. O’Boy, V.V. Krylov and J.L. Horner, "Effect of geometrical and material imperfections on damping flexural vibrations in plates with attached wedges of power law profile", Applied Acoustics, vol. 73, pp. 514-523, 2012. 
[26] J.J. Bayod, "Experimental study of vibration damping in a modified elastic wedge of power-law profile", Journal of Vibration and Acoustics, vol. 133, 061003, 2011.

[27] D.J. O’Boy and V.V. Krylov, "Damping of flexural vibrations in circular plates with tapered central holes, Journal of Sound and Vibration", vol. 330, pp. 2220-2236, 2011.

[28] D.J. O’Boy, E.P. Bowyer and V.V. Krylov, "Point mobility of a cylindrical plate incorporating a tapered hole of power-law profile", J. Acoust. Soc. Amer., vol. 129(6), pp. 3475-3482, 2011.

[29] E.P. Bowyer, D.J. O'Boy, V.V. Krylov and F. Gautier, "Experimental investigation of damping flexural vibrations in plates containing tapered indentations of power-law profile", Applied Acoustics, 74(4), pp. 553-560, 2013.

[30] E.P. Bowyer and V.V. Krylov, "Sound radiation of rectangular plates containing tapered indentations of power-law profile", Proceedings of Meetings on Acoustics (POMA), vol. 18, pp. 030002-13, 2013.

[31] E.P. Bowyer, V.V. Krylov and D.J. O’Boy, "Damping of flexural vibrations in rectangular plates by slots of power-law profile", Proceedings of the Conference ‘Acoustics 2012’, Nantes, France, 23-27 April 2012, pp. 2187-2192.

[32] E.P. Bowyer, J.M. Lister, V.V. Krylov and D.J. O’Boy, "Experimental study of damping flexural vibrations in tapered turbofan blades", Proceedings of the Conference ‘Acoustics 2012’, Nantes, France, 23-27 April 2012, pp. 2201- 2206.

[33] E.P. Bowyer and V.V. Krylov, "Damping of flexural vibrations in turbofan blades using the acoustic black hole effect", Applied Acoustics, vol. 76, pp. 359-365, 2014.

[34] E.P. Bowyer, P. Nash and V.V. Krylov, "Damping of flexural vibrations in glass fibre composite plates and honeycomb sandwich panels containing indentations of power-law profile", Proceedings of Meetings on Acoustics (POMA), vol. 18, pp.030004-13, 2013. 
[35] E.P. Bowyer and V.V. Krylov, "Experimental investigation of damping flexural vibrations in glass fibre composite plates containing one- and two-dimensional acoustic black holes", Composite Structures, vol. 107, pp. 406-415, 2014.

[36] M.A. Mironov and V.V. Pislyakov, "One-dimensional acoustic waves in retarding structures with propagation velocity tending to zero", Acoustical Physics, vol. 48 (3), pp. 347-352, 2002.

[37] O. Umnova and B. Zajamsek, "Omnidirectional graded index sound absorber", Proceedings of the Conference 'Acoustics 2012', Nantes, France, 23-27 April 2012, pp. 3631-3637.

[38] M.A. Isakovich, General Acoustics, Nauka, Moscow, 1973 (in Russian).

[39] M.C. Junger and D. Feit, Sound, Structures and their Interaction, 2nd Edition, MIT Press, Cambridge, MA, 1986.

[40] W.G. Unruh, “Experimental black hole evaporation?”, Physical Review Letters, vol. 46, pp. 1351-1353, 1981.

[41] W.G. Unruh, "Sonic analogue of black holes and the effect of high frequencies on black hole evaporation”, Physical Review D, vol. 51, pp. 2827-2838, 1995.

[42] M. Visser, “Acoustic black holes: horizons, ergospheres and Hawking radiation”, Classical and Quantum Gravity, vol. 15, pp. 1767-1791, 1998.

[43] O. Lahav, A. Itah, A. Blumkin, C. Gordon, S. Rinott, A. Zayats and J. Steinhauer, “ Realisation of a sonic black hole analog in a Bose-Einstein condensate”, Physical Review Letters, vol. 105, pp. 240401-4, 2010. 